\title{
Cognitive deficits in Progressive Supranuclear Palsy on the Repeatable Battery for the Assessment of Neuropsychological Status
}

\author{
Kevin Duff ${ }^{1}$, Dana McDermott ${ }^{2}$, Dan Luong ${ }^{2}$, Christopher Randolph ${ }^{3}$, and Adam L. Boxer ${ }^{2}$ \\ on behalf of the AL-108-213 investigators \\ 1Department of Neurology, University of Utah, Salt Lake City, UT \\ ${ }^{2}$ Memory and Aging Center, Department of Neurology, University of California, San Francisco, CA \\ ${ }^{3}$ Department of Neurology, Loyola University Medical Center, Maywood, IL
}

\section{Abstract}

Objective: Progressive supranuclear palsy (PSP) is associated with a variety of cognitive deficits, as well as motor and psychiatric disturbances. As clinical trials for PSP evolve, briefer screening instruments will be needed to determine cognitive effects of interventions. The Repeatable Battery for the Assessment of Neuropsychological Status (RBANS) may fill this gap.

Methods: Three hundred four participants diagnosed with Richardson's syndrome of PSP were evaluated with the RBANS, as well as other scales typically used in PSP.

Results: RBANS performances for these participants fell significantly below expectations for the Total Scale score and all five Indexes. Cognitive scores on the RBANS were also significantly related to other markers of PSP (e.g., motor and functional abilities, depression, global cognition). Compared to other clinical conditions from the literature, patients with PSP show impairment on tests of visuospatial perception and construction and attention.

Conclusion: Although additional research is needed, the current study supports the clinical applicability of the RBANS in patients with PSP, as well as its potential for future clinical trials.

\section{Keywords}

progressive supranuclear palsy; cognition; neurological disease; dementia; RBANS

\section{Introduction}

Progressive supranuclear palsy (PSP) is a rare neurodegenerative condition, which is characterized by abnormal aggregates of the microtubule-associated protein tau in neurons and glia throughout the brain, but most prominently in the brainstem and basal ganglia (Boxer et al., 2017). Although most cases are sporadic, PSP can have an autosomal

\footnotetext{
Address correspondences to Kevin Duff, PhD, Center for Alzheimer's Care, Imaging and Research, Department of Neurology, University of Utah, 650 Komas Drive \#106-A, Salt Lake City, UT 84108. Tel: 801-585-9983. Fax: 801-581-2483.

kevin.duff@hsc.utah.edu.
} 
dominant pattern of inheritance, and it is associated with mutations of the MAPT gene on chromosome 17. Clinically, Richardson's syndrome of PSP (Hoglinger, Respondek, et al., 2017) presents with a variety of motor disturbances (e.g., early and severe gait instability, vertical gaze palsy, rigidity of the axial muscles, dysphagia, pseudobulbar affect) (Litvan \& Hutton, 1998), neuropsychiatric symptoms (e.g., apathy, depression, sleep disturbances, agitation, disinhibition) (Gerstenecker, Duff, Mast, Litvan, \& Group, 2013), and cognitive impairment. This triad of clinical symptoms often leads to notable functional deficits (K. Duff, Gerstenecker, \& Litvan, 2013).

A wide range of cognitive difficulties are seen in PSP, including those adversely affecting phonemic and semantic verbal and figural fluency (Bak, Crawford, Hearn, Mathuranath, \& Hodges, 2005; Grafman et al., 1990; Soliveri et al., 2000), naming (Cotelli et al., 2006), attention and executive functioning (Bak et al., 2005; Gerstenecker, Mast, et al., 2013; Paviour et al., 2005), and learning and memory (Litvan, 1994). Despite multiple cognitive domains being affected, Gerstenecker et al. (2013) found primary executive dysfunction (e.g., initiating and inhibiting responses, especially on tasks with a motor output) in a large cohort of these patients. As the disease progresses and these patients experience progressive cognitive decline, it may become difficult to comprehensively evaluate their cognitive functioning with lengthy batteries and briefer screening measures of cognitive abilities are needed.

The Repeatable Battery for the Assessment of Neurological Status (RBANS) (Randolph, 1998) is a brief cognitive screening battery that has been used to evaluate neuropsychological functioning in a wide range of neuropsychiatric conditions. To our knowledge, only two studies have used this battery to evaluate patients with PSP. Boxer et al. (2014) did not find a treatment effect in 313 patients with PSP on the RBANS Total Scale score in a clinical trial comparing davunetide and placebo across 52 weeks. Using the same sample from this clinical trial, Bang et al. (2016) reported that the Total Sale score on the RBANS was associated with attrition in the trial and with changes in symptoms of PSP across the year-long study. These studies provide preliminary support for the RBANS in clinical trials in PSP, but they only focused on the Total Scale score of the RBANS, which limits this battery's utility in defining the cognitive deficits in PSP.

Therefore, the purpose of the present study was to further validate the RBANS as a tool to assess cognitive functioning in a large, well-characterized sample of patients with PSP. In addition, relationships between RBANS scores and other markers of PSP will be examined. Finally, comparisons will be made to other relevant clinical studies in the literature that used the RBANS. As clinical trials in PSP expand, a well-validated cognitive measure could be useful in determining the effectiveness of treatments.

\section{Methods}

\section{Participants and Procedures}

Participants were recruited from 48 study centers in Australia, Canada, France, Germany, the United Kingdom, and the United States for a randomized clinical trial of davunetide vs. placebo (Boxer et al., 2014). Ethics board approval was obtained at each site and all 
participants gave written informed consent as per local regulations. All participants met the following inclusion criteria for PSP: at least a 12-month history of postural instability or falls, decreased downward saccade velocity or supranuclear ophthalmoplegia, and an akinetic-rigid syndrome with prominent axial rigidity. In addition, at screening, individuals had to be between 41 to 85 years old, have a Mini-Mental State Examination (MMSE) score $\geq 15$, live outside a skilled nursing facility or dementia care facility, be able to ambulate independently or to take at least 5 steps with minimal assistance, have a Progressive Supranuclear Palsy Rating Scale (PSPRS) score $\geq 40$, and be able to undergo an MRI scan during screening. Participants were allowed to take Parkinson's medications if the dose had been stable for 60 days prior to screening. Participants were allowed to take rasagiline or coenzyme Q10 if the dose was stable for at least 90 days prior to screening. Participants were excluded if: they had a clear and robust benefit from levodopa at the time of screening, evidence of motor neuron disease, or use of acetylcholinesterase inhibitors, antipsychotics (other than quetiapine), memantine, lithium, methylene blue, or other putative disease modifying drugs for PSP. These were the same participants used in other papers that reported on the RBANS Total Scale score (Bang et al., 2016; Boxer et al., 2014), but these other papers have not provided information on the other Indexes of the RBANS.

\section{Measures}

The RBANS (Randolph, 1998) is a brief, individually administered test measuring attention, language, visuospatial/constructional abilities, and immediate and delayed memory. It consists of twelve subtests, which yield five Index scores and a Total Scale score. Normative information from the manual, which is used to calculate the Index and Total scores, is based on 540 healthy adults who ranged in age from 20-89 years old. The Index and Total scores are age-corrected standard scores $(\mathrm{M}=100, \mathrm{SD}=15)$. All subtests were administered and scored as defined in the manual. Validated translations of the RBANS were used in this study, and all participants were tested in their primary language.

In addition to the RBANS, a number of other commonly-used measures in PSP were collected on these individuals, including:

- $\quad$ The PSPRS (Golbe \& Ohman-Strickland, 2007) is a widely-used rating scale for symptoms of PSP, which consists of six categories including daily activities, behavior, bulbar, oculomotor, limb motor, and gait/midline. Scores range from 0 to 100 , with higher scores indicating more severe disease.

- $\quad$ The Schwab and England Activities of Daily Living (SEADL) (Schwab \& England, 1969) scale is a measure of overall disability based on interviews with the patient and the informant. It is scored on an ordinal scale, with 0.0 indicating vegetative functions, up to 1.0 indicating complete independence.

- $\quad$ The Geriatric Depression Scale (GDS) (Yesavage et al., 1983) is a 30-item screening measure for symptoms of depression in the elderly. Higher scores indicate more severe depression. 
- $\quad$ The MMSE (Folstein, Folstein, \& McHugh, 1975) is a widely-used screening measure for global cognitive functioning. Higher scores indicate better cognitive functioning.

Boxer et al. (2014) previously presented results from this cohort on three tests of executive functioning (Color Trails, Letter-Number Sequencing, and phonemic fluency). We elected not to re-present those findings, as: 1) we wanted to focus on the RBANS profile of PSP, and 2) comparisons between measures would be difficult without a common normative database. Finally, not all participants completed all tests due to time restraints, participant fatigue, or severity of cognitive impairments, and only participants with all measures were included.

\section{Data Analyses}

Primary analyses.-To validate the utility of the RBANS in those with PSP, one sample t-tests were used to compare Index scores of the participants with PSP to an expected value of 100 (i.e., age-corrected score at the $50^{\text {th }}$ percentile). Cohen's d, a measure of effect size, was calculated from the respective means and standard deviations. The assumptions of a one-sample t-test were met (i.e., RBANS data were continuous, RBANS data followed a normal distribution [e.g., all skewness and kurtosis values were less than 3], sample is randomly drawn from its population). Pearson correlations were calculated between RBANS scores and other scale frequently used in patients with PSP (e.g., PSPRS, SEADL, GDS, MMSE). An alpha of 0.05 was used throughout.

Secondary analyses.-Since no control data are presented, comparisons were made between the RBANS performances of the current PSP sample and other relevant clinical studies in the existing literature. Specifically, the means and standard deviations for patients with Alzheimer's disease (AD, $n=69$, recruited from southern US medical center's memory disorders clinic, MMSE: $\mathrm{M}=22.4, \mathrm{SD}=3.9$ ) (K. Duff et al., 2008), Parkinson's disease with dementia ( $\mathrm{PD}, \mathrm{n}=27$, recruited from Oklahoma City medical center's movement disorders clinic, MMSE: $\mathrm{M}=23.1, \mathrm{SD}=2.5$ ) (Beatty et al., 2003), and Huntington's disease (HD, $\mathrm{n}=75$, recruited from University of Iowa's Huntington's Disease Center of Excellence, no MMSE data on this sample) (K Duff, Beglinger, Theriault, Allison, \& Paulsen, 2010) were compared with our PSP patients with one-sample t-tests. Effect sizes were also estimated. Additional details about these other clinical samples can be obtained from the original articles.

\section{Results}

\section{Primary analyses}

Three hundred four participants PSP participants were included in the final sample. They were nearly evenly distributed for gender (53\% male and $47 \%$ female), with a mean age of $67.6(\mathrm{SD}=6.5)$ years. The vast majority was white $(88 \%)$ and not Hispanic $(91 \%)$. Disease duration was reported to be fewer than five years for $91 \%$ of the sample, and more than five years for $9 \%$. Based on their PSPRS score, they tended to be mildly to moderately impaired $(\mathrm{M}=39.9, \mathrm{SD}=11.1)$. Daily functioning tended to show increasing dependence (SEADL: $\mathrm{M}=50, \mathrm{SD}=20$ ). Overall, they were mildly depressed (GDS: $\mathrm{M}=12.7, \mathrm{SD}=6.7$ ), with 
borderline cognition (MMSE: $\mathrm{M}=26.2, \mathrm{SD}=3.6$ ). See Table 1 for RBANS Index scores, which ranged from moderately impaired to borderline.

One sample t-tests for the RBANS Indexes indicated that all fell significantly below 100 (see Table 1), with effect sizes (i.e., Cohen's d) for all RBANS Indexes being large to very large. The largest discrepancies were seen on the Visuospatial/Constructional Index $(d=5.3)$. Large effect sizes were also seen on the Attention and Language Indexes $(\mathrm{d}=3.4$ and 3.0, respectively).

Relationships between the RBANS Indexes and other markers of PSP are presented in Table 2. Briefly, all of these important clinical characteristics of PSP were significantly correlated with the Total Scale score of the RBANS, including PSPRS $(r=-0.41, p<0.001)$, SEADL $(r=0.45, p<0.001)$, GDS $(r=-0.24, p<0.001)$, and MMSE $(r=0.65, p<0.001)$. Similar relationships existed between the RBANS Indexes and these markers of PSP.

\section{Secondary analyses}

The means and standard deviations of the RBANS Indexes for the PSP sample are reiterated in Table 3, as are the RBANS scores for the samples of $\mathrm{AD}, \mathrm{PD}$, and HD. Compared to patients with $\mathrm{AD}$, those with PSP performed significantly poorer on the Visuospatial/ Constructional Index $(p<0.001, d=2.7)$ and the Attention Index $(p<0.001, d=0.6)$, whereas patients with AD performed significantly worse on the Immediate Memory Index ( $p$ $<0.001, d=2.4)$, Language Index ( $<0.001, d=0.5)$, Delayed Memory Index $(\mathrm{p}<0.001, \mathrm{~d}$ $=3.7)$, and Total Scale $(\mathrm{p}<0.001, \mathrm{~d}=1.5)$. These cognitive profiles are presented in Figure 1.

Using the sample of patients with PD with dementia from Beatty et al. (2003), PSP patients performed significantly worse on the Visuospatial/Constructional Index $(p<0.01, d=0.6)$ and the Language Index $(\mathrm{p}<0.001, \mathrm{~d}=0.9)$, whereas the patients with PD and dementia performed significantly worse on the Immediate Memory Index $(p<0.001, d=3.1)$, Attention Index $(\mathrm{p}<0.001, \mathrm{~d}=1.1)$, Delayed Memory Index $(\mathrm{p}<0.001, \mathrm{~d}=2.4)$, and Total Scale $(\mathrm{p}<0.001, \mathrm{~d}=1.9)$. These cognitive profiles are presented in Figure 2.

Compared to the HD patients in Duff et al. (2009), the PSP patients of the current sample performed significantly better on most of the RBANS Indexes (Immediate Memory [p $<0.001, \mathrm{~d}=1.5$ ], Attention [p $<0.001, \mathrm{~d}=0.9$ ], Delayed Memory [p $<0.001, \mathrm{~d}=1.1$ ], Total Scale $[\mathrm{p}<0.001, \mathrm{~d}=0.5])$. The HD patients performed significantly better on the Visuospatial Constructional Index ( $p<0.01, \mathrm{~d}=1.8$ ], and the two groups were comparable on the Language Index $(\mathrm{p}=0.07)$. These cognitive profiles are also presented in Figure 2.

\section{Discussion}

The current study sought to further validate the RBANS as a tool to assess cognitive functioning in PSP, for which motor, psychiatric, and cognitive disturbances are common. As clinical trials in PSP expand, a well-validated cognitive measure could be useful in determining the effectiveness of treatments, and the RBANS may serve this unique role. Consistent with prior studies (Bak et al., 2005; Gerstenecker, Mast, et al., 2013; Litvan, 
1994), significant cognitive deficits were observed in this cohort of patients with PSP. For example, as seen in two prior studies using the RBANS in PSP (Bang et al., 2016; Boxer et al., 2014), the mean Total Scale score for this sample fell at the $4^{\text {th }}$ percentile. This similar result is not surprising, as we used the same sample as these prior studies. However, unlike these prior studies, we were also able to examine the five Indexes of the RBANS. Across these other cognitive domains, the patients with PSP performed the worst on Indexes tapping visuospatial perception and construction (Visuospatial/Constructional Index: $\mathrm{M}=68.9, \mathrm{SD}=$ 11.8) and attention (Attention Index: $\mathrm{M}=74.0, \mathrm{SD}=15.2$ ). Compared to their other cognitive scores, learning and memory and language tended to be more intact in these patients. Such a pattern of test scores across cognitive domains would tend to fit with a "subcortical" profile. Indeed, using the Cortical Subcortical Index of Randolph et al. (Randolph, Tierney, Mohr, \& Chase, 1998), this sample of patients with PSP had a decidedly "subcortical" cognitive profile on the RBANS (M $=-11$, where scores $₫)$ indicate a subcortical profile).

Despite the significant deficits on the RBANS by patients with PSP, the current data cannot determine if this reflects a true "cognitive footprint" of this condition or if this reflects a more general neuropsychological profile of patients with a variety of neurodegenerative disorders. The secondary analyses in the current study can only begin to address this question. First, the patients with PSP did worse than all of the other clinical groups on the Visuospatial/Constructional Index (e.g., 14 Index points worse than the patients with AD, 10 Index points worse than the patients with HD, 2 Index points worse than the patients with PD with dementia). Second, the patients with PSP tended to perform significantly better than the other clinical groups on the Immediate Memory (20 Index points higher than patients with AD, 17 Index points higher than patients with PD with dementia, 13 Index points higher than patients with HD) and Delayed Memory Indexes (28 Index points higher than patients with $\mathrm{AD}, 12$ Index points higher than patients with PD with dementia, 9 Index points higher than patients with HD). Third, for the Attention Index, there was more intergroup differences. Patients with PSP performed slightly better than those with PD with dementia (5 Index points) and those with HD (6 Index points), but they performed slightly worse than those with AD (4 Index points). Fourth, the PSP patients performed relatively comparably to the other clinical groups on the Language Index ( +2 Index points compared to $\mathrm{AD},-3$ Index points compared to $\mathrm{PD}$ with dementia, +1 Index points compared to HD). Although there are clearly other neurological and psychiatric signs and symptoms that separate these clinical disorders, they all share cognitive difficulties, and comparisons of their cognitive profiles on a common measure like the RBANS can be informative.

As with other neuropsychiatric disorders, the presentation of Richardson's syndrome phenotype of PSP varies considerably between individuals. Some individuals present with more noticeable motor dysfunction, whereas others present with more cognitive or psychiatric disturbances. It is informative to see how these symptoms of PSP co-occur. As observed in the correlational analyses, the cognitive difficulties observed on the RBANS appear to vary with the motor abnormalities and functional declines in patients with PSP. For example, significant correlations between all RBANS scores and the PSPRS and SEADL were noted, with worse cognition being associated with more PSP-related disability. Although slightly smaller relationships, it is also interesting to note that RBANS 
performances varied with depressive symptoms, as indicated by the GDS. Again, more depressive symptoms were associated with worse cognition, which is consistent with the literature in PSP (Gerstenecker, Duff, et al., 2013). Finally, a global measure of cognition (MMSE) was positively correlated with all of the RBANS Indexes. So, if the RBANS is to be considered for future clinical trials in PSP, researchers should be encouraged that it captures some shared variance with other characteristic symptoms of PSP. It also possesses other important features of adequate neurocognitive endpoints, such as the availability of alternate forms (e.g., four forms available through test publisher), brevity of administration, and availability of translations for multinational studies (e.g., translated into over 20 languages).

Despite the supportive findings for using the RBANS in patients with PSP, some limitations should be mentioned. First, we did not utilize a control group in the current study, and future studies should attempt to find appropriate comparison participants. Although not ideal, comparing these patients to expectations based on age-matched normative data are more analogous to clinical practice. Education was also not collected in this study, which is another important variable when considering cognitive functioning, particularly in such a diverse sample. The lack of a control group also prevents the calculation of sensitivity and specificity values for the RBANS in our PSP sample. Our use of three "historical" comparator groups (AD, PD, HD) had similar limitations (e.g., no control of education, sex, or race/ethnicity; unable to calculate sensitivity/specificity; unable to conduct more sophisticated analyses [e.g., profile analysis]), which may have influenced the results. Second, subtest scores for the RBANS were not available for analysis. It is likely that the subtests that make up the Index scores would provide a more fruitful examination of the cognitive difficulties associated with PSP. Third, the current study used a cohort of patient who were relatively early in the course of their disease (e.g., $91 \%$ had a disease duration of $<5$ years, inclusion criteria included MMSE score 215 ). In this way, our results likely apply to an early "window" of PSP, rather than the entire spectrum. Fourth, there are other biomarkers of PSP that were not available for analysis. For example, future studies might also examine the relationship between the RBANS and volumetric neuroimaging or neurofilament light chain or phosphorylated tau (Dutt et al., 2016; Hoglinger, Schope, et al., 2017; Rojas et al., 2018). Fifth, the RBANS was developed as a screening battery, and more comprehensive neuropsychological batteries will likely detect even more subtle cognitive difficulties in PSP. The RBANS also does not include measures of executive functioning, for which deficits are frequently seen in PSP (Gerstenecker, Mast, et al., 2013). Although we elected not to re-present the results of three non-RBANS tests of executive functioning in this cohort, they can be found in Boxer et al. (2014). Finally, despite using validated translations of the RBANS and testing participants in their primary language, there are many inherent challenges with conducting multi-national clinical trials. The RBANS has been used in many samples outside of the United States (Baune et al., 2010; Collinson et al., 2014; De la Torre et al., 2014; Goldbecker et al., 2013; Liogier d'Ardhuy et al., 2015; Yamashima et al., 2002), but continued work on examining the effects on test administration, scoring, and norming is needed. Regardless of these limitations, the current study provides additional support for using the RBANS to assess cognition in patients with PSP, and future studies might extend these findings with longitudinal comparisons of PSP patients. 


\section{Acknowledgements:}

The project described was supported by research grants from the NIH (U54NS092089 and R01AG038791), the Tau Consortium, and the UCSF Parkinson's Spectrum Disorder Center.

\section{References}

Bak TH, Crawford LM, Hearn VC, Mathuranath PS, \& Hodges JR (2005). Subcortical dementia revisited: similarities and differences in cognitive function between progressive supranuclear palsy (PSP), corticobasal degeneration (CBD) and multiple system atrophy (MSA). Neurocase, 11(4), 268-273. doi: 10.1080/13554790590962997 [PubMed: 16093227]

Bang J, Lobach IV, Lang AE, Grossman M, Knopman DS, Miller BL, ... Investigators, A. L. (2016). Predicting disease progression in progressive supranuclear palsy in multicenter clinical trials. Parkinsonism Relat Disord, 28, 41-48. doi: 10.1016/j.parkreldis.2016.04.014 [PubMed: 27172829]

Baune BT, Miller R, McAfoose J, Johnson M, Quirk F, Mitchell D. The role of cognitive impairment in general functioning in major depression. Psychiatry Res 20104 30;176(2-3):183-9. doi: 10.1016/j.psychres.2008.12.001. [PubMed: 20138370]

Beatty WW, Ryder KA, Gontkovsky ST, Scott JG, McSwan KL, \& Bharucha KJ (2003). Analyzing the subcortical dementia syndrome of Parkinson's disease using the RBANS. Arch Clin Neuropsychol, 18(5), 509-520. [PubMed: 14591446]

Boxer AL, Lang AE, Grossman M, Knopman DS, Miller BL, Schneider LS, ... Investigators, A. L. (2014). Davunetide in patients with progressive supranuclear palsy: a randomised, double-blind, placebo-controlled phase 2/3 trial. Lancet Neurol, 13(7), 676-685. doi: 10.1016/ S1474-4422(14)70088-2 [PubMed: 24873720]

Boxer AL, Yu JT, Golbe LI, Litvan I, Lang AE, \& Hoglinger GU (2017). Advances in progressive supranuclear palsy: new diagnostic criteria, biomarkers, and therapeutic approaches. Lancet Neurol, 16(7), 552-563. doi: 10.1016/S1474-4422(17)30157-6 [PubMed: 28653647]

Collinson SL, Fang SH, Lim ML, Feng L, Ng TP. Normative data for the repeatable battery for the assessment of neuropsychological status in elderly Chinese. Arch Clin Neuropsychol 2014 8;29(5): 442-55. doi: 10.1093/arclin/acu023. [PubMed: 24903208]

Cotelli M, Borroni B, Manenti R, Alberici A, Calabria M, Agosti C, ... Cappa SF (2006). Action and object naming in frontotemporal dementia, progressive supranuclear palsy, and corticobasal degeneration. Neuropsychology, 20(5), 558-565. doi: 10.1037/0894-4105.20.5.558 [PubMed: 16938018]

De la Torre GG, Suárez-Llorens A, Caballero FJ, Ramallo MA, Randolph C, Lleó A, Sala I, Sánchez B. Norms and reliability for the Spanish version of the Repeatable Battery for the Assessment of Neuropsychological Status (RBANS) Form A. J Clin Exp Neuropsychol 2014;36(10):1023-30. doi: 10.1080/13803395.2014.965664. [PubMed: 25363544]

Duff K, Beglinger L, Theriault D, Allison J, \& Paulsen J (2010). Cognitive deficits in Huntington's disease on the Repeatable Battery for the Assessment of Neuropsychological Status. J Clin Exp Neuropsychol, 32(3), 231-238. [PubMed: 19484645]

Duff K, Gerstenecker A, \& Litvan I (2013). Functional impairment in progressive supranuclear palsy. Neurology, 80(4), 380-384. [PubMed: 23303854]

Duff K, Humphreys Clark JD, O’Bryant SE, Mold JW, Schiffer RB, \& Sutker PB (2008). Utility of the RBANS in detecting cognitive impairment associated with Alzheimer's disease: sensitivity, specificity, and positive and negative predictive powers. Arch Clin Neuropsychol, 23(5), 603-612. [PubMed: 18639437]

Dutt S, Binney RJ, Heuer HW, Luong P, Attygalle S, Bhatt P, ... investigators, A. L. (2016). Progression of brain atrophy in PSP and CBS over 6 months and 1 year. Neurology, 87(19), 20162025. doi: 10.1212/WNL.0000000000003305 [PubMed: 27742814]

Folstein MF, Folstein SE, \& McHugh PR (1975). "Mini-mental state". A practical method for grading the cognitive state of patients for the clinician. J Psychiatr Res, 12(3), 189-198. [PubMed: 1202204] 
Gerstenecker A, Duff K, Mast B, Litvan I, \& Group, E.-P. S. (2013). Behavioral abnormalities in progressive supranuclear palsy. Psychiatry Res, 210(3), 1205-1210. doi: 10.1016/j.psychres. 2013.08.045 [PubMed: 24035530]

Gerstenecker A, Mast B, Duff K, Ferman TJ, Litvan I, \& Group E-PS (2013). Executive dysfunction is the primary cognitive impairment in progressive supranuclear palsy. Arch Clin Neuropsychol, 28(2), 104-113. doi: 10.1093/arclin/acs098 [PubMed: 23127882]

Golbe LI, \& Ohman-Strickland PA (2007). A clinical rating scale for progressive supranuclear palsy. Brain, 130(Pt 6), 1552-1565. [PubMed: 17405767]

Goldbecker A, Weissenborn K, Hamidi Shahrezaei G, Afshar K, Rümke S, Barg-Hock H, Strassburg $\mathrm{CP}$, Hecker H, Tryc AB. Comparison of the most favoured methods for the diagnosis of hepatic encephalopathy in liver transplantation candidates. Gut 2013 10;62(10):1497-504. doi: 10.1136/ gutjnl-2012-303262. [PubMed: 23297006]

Grafman J, Weingartner H, Newhouse PA, Thompson K, Lalonde F, Litvan I, ... Sunderland T (1990). Implicit learning in patients with Alzheimer's disease. Pharmacopsychiatry, 23(2), 94-101. doi: $10.1055 / \mathrm{s}-2007-1014490$

Hoglinger GU, Respondek G, Stamelou M, Kurz C, Josephs KA, Lang AE, ... Movement Disorder Society-endorsed, P. S. P. S. G. (2017). Clinical diagnosis of progressive supranuclear palsy: The movement disorder society criteria. Mov Disord, 32(6), 853-864. doi: 10.1002/mds.26987 [PubMed: 28467028]

Hoglinger GU, Schope J, Stamelou M, Kassubek J, Del Ser T, Boxer AL, ... Movement Disorder Society-Endorsed, P. S. P. S. G. (2017). Longitudinal magnetic resonance imaging in progressive supranuclear palsy: A new combined score for clinical trials. Mov Disord, 32(6), 842-852. doi: 10.1002/mds.26973 [PubMed: 28436538]

Liogier d'Ardhuy X, Edgin JO, Bouis C, de Sola S, Goeldner C, Kishnani P, Nöldeke J, Rice S, Sacco S, Squassante L, Spiridigliozzi G, Visootsak J, Heller J, Khwaja O. Assessment of Cognitive Scales to Examine Memory, Executive Function and Language in Individuals with Down Syndrome: Implications of a 6-month Observational Study. Front Behav Neurosci 201511 18;9:300. doi: 10.3389/fnbeh.2015.00300. eCollection 2015. [PubMed: 26635554]

Litvan I (1994). Cognitive disturbances in progressive supranuclear palsy. J Neural Transm Suppl, 42, 69-78. [PubMed: 7964698]

Litvan I, \& Hutton M (1998). Clinical and genetic aspects of progressive supranuclear palsy. J Geriatr Psychiatry Neurol, 11(2), 107-114. [PubMed: 9877531]

Paviour DC, Winterburn D, Simmonds S, Burgess G, Wilkinson L, Fox NC, ... Jahanshahi M (2005). Can the frontal assessment battery (FAB) differentiate bradykinetic rigid syndromes? Relation of the FAB to formal neuropsychological testing. Neurocase, 11(4), 274-282. doi: 10.1080/13554790590962933 [PubMed: 16093228]

Randolph C (1998). Repeatable Battery for the Assessment of Neuropsychological Status San Antonio, TX: The Psychological Coporation.

Randolph C, Tierney MC, Mohr E, \& Chase TN (1998). The Repeatable Battery for the Assessment of Neuropsychological Status (RBANS): preliminary clinical validity. J Clin Exp Neuropsychol, 20(3), 310-319. [PubMed: 9845158]

Rojas JC, Bang J, Lobach IV, Tsai RM, Rabinovici GD, Miller BL, ... Investigators, A. L. (2018). CSF neurofilament light chain and phosphorylated tau 181 predict disease progression in PSP. Neurology, 90(4), e273-e281. doi: 10.1212/WNL.0000000000004859 [PubMed: 29282336]

Schwab RS, \& England AC (1969). Projection technique for evaluating surgery in Parkinson's disease. In Gillingham FJ \& Donaldson MC (Eds.), Third Symposium on Parkinson's Disease (pp. 152157). Edinburgh: Livingstone.

Soliveri P, Monza D, Paridi D, Carella F, Genitrini S, Testa D, \& Girotti F (2000). Neuropsychological follow up in patients with Parkinson's disease, striatonigral degeneration-type multisystem atrophy, and progressive supranuclear palsy. J Neurol Neurosurg Psychiatry, 69(3), 313-318. [PubMed: 10945805]

Yamashima T, Yoshida M, Kumahashi K, Matsui M, Koshino Y, Higashima M, Nagasawa T, Ueki A, Ohtsuka M, Aoki S, Imuro S, Mori N, Takei N, Hoshino R, Minabe Y, Nanba Y, Nanba M, Kira J, Ohyagi Y, Haraoka J, Akimoto J, Miura N, Kimura S, Matsushita M. [The Japanese version of 
RBANS (Repeatable Battery for the Assessment of Neuropsychological Status)]. No To Shinkei 2002 6;54(6):463-71. Japanese. [PubMed: 12166095]

Yesavage JA, Brink TL, Rose TL, Lum O, Huang V, Adey MB, \& Leirer VO (1983). Development and validation of a geriatric depression screening scale: A preliminary report. Journal of Psychiatric Research, 17, 37-49. 


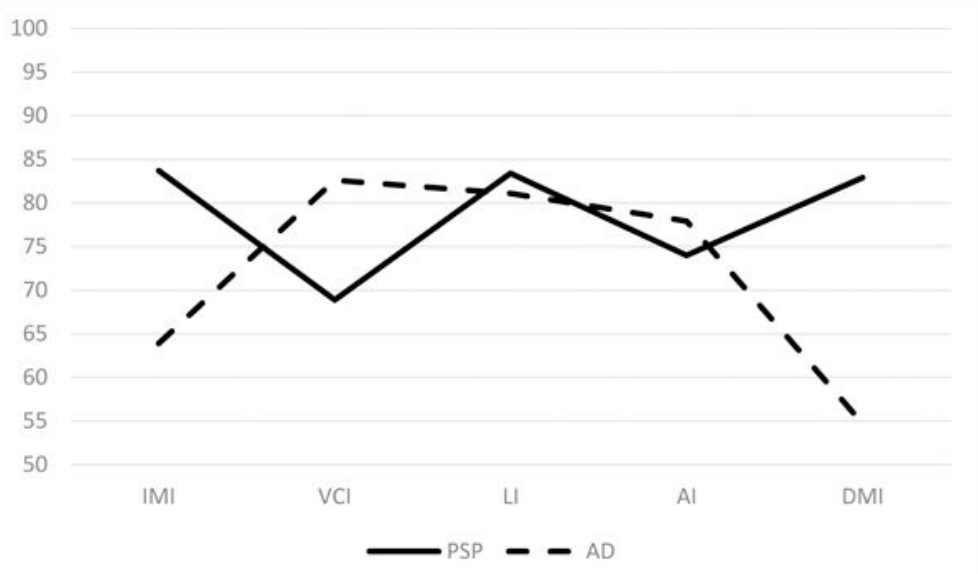

Note. $y$-axis = age-corrected standard scores $(M=100, S D=15) . X$-axis $=$ RBANS Indexes. IMI $=$ Immediate Memory Index, VCI = Visuospatial/Constructional Index, $\mathrm{LI}=$ Language Index, $\mathrm{Al}=$ Attention Index, $\mathrm{DMI}=$ Delayed Memory Index, $\mathrm{PSP}=$ progressive supranuclear palsy, $A D=$ Alzheimer's disease .

Figure 1.

RBANS profiles of PSP and AD. 


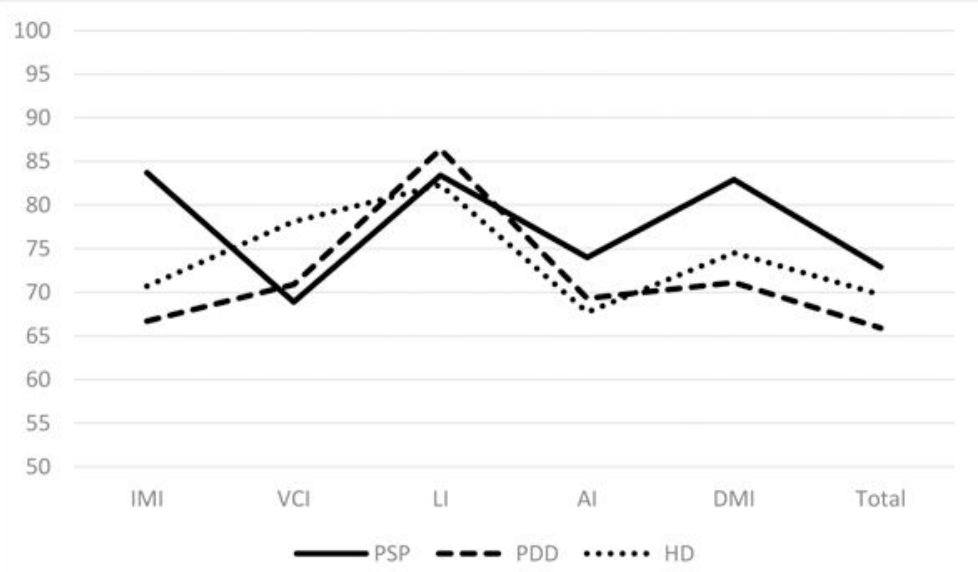

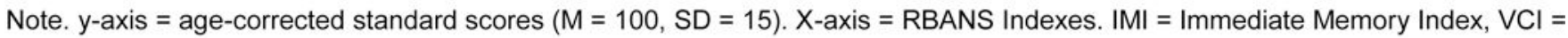
Visuospatial/Constructional Index, $\mathrm{LI}=$ Language Index, $\mathrm{Al}=$ Attention Index, $\mathrm{DMI}=$ Delayed Memory Index, $\mathrm{PSP}=$ progressive supranuclear palsy, $\mathrm{PDD}=$ Parkinson's disease with dementia, $\mathrm{HD}=$ Huntington's disease.

Figure 2.

RBANS profiles of PSP, PD with dementia, and HD. 


\section{Table 1}

RBANS Indexes in the PSP sample

\begin{tabular}{lcccc}
\hline RBANS Indexes & Mean (SD) & Range & $\mathbf{t}(\mathbf{d f}) \mathbf{p}$ & $\mathbf{d}$ \\
\hline Immediate Memory & $83.7(19.3)$ & $44-126$ & $-14.8(303)<0.001$ & 1.7 \\
Visuospatial/Constructional & $68.9(11.8)$ & $50-112$ & $-45.9(303)<0.001$ & 5.3 \\
Language & $83.4(10.9)$ & $51-134$ & $-26.4(303)<0.001$ & 3.0 \\
Attention & $74.0(15.2)$ & $43-118$ & $-29.8(303)<0.001$ & 3.4 \\
Delayed Memory & $82.9(17.6)$ & $40-117$ & $-16.9(303)<0.001$ & 1.9 \\
Total Scale & $72.9(13.1)$ & $44-105$ & $-36.1(303)<0.001$ & 4.1 \\
\hline
\end{tabular}

Note. Index scores are age-corrected using the standardization sample from the RBANS manual. Means and standard deviations (in parentheses) are presented in the second column. $\mathrm{t}=\mathrm{t}$-value from one sample $\mathrm{t}$-test. $\mathrm{df}=$ degrees of freedom. $\mathrm{p}=\mathrm{p}$-value. $\mathrm{d}=\mathrm{C}$ Chen's $\mathrm{d}$. 


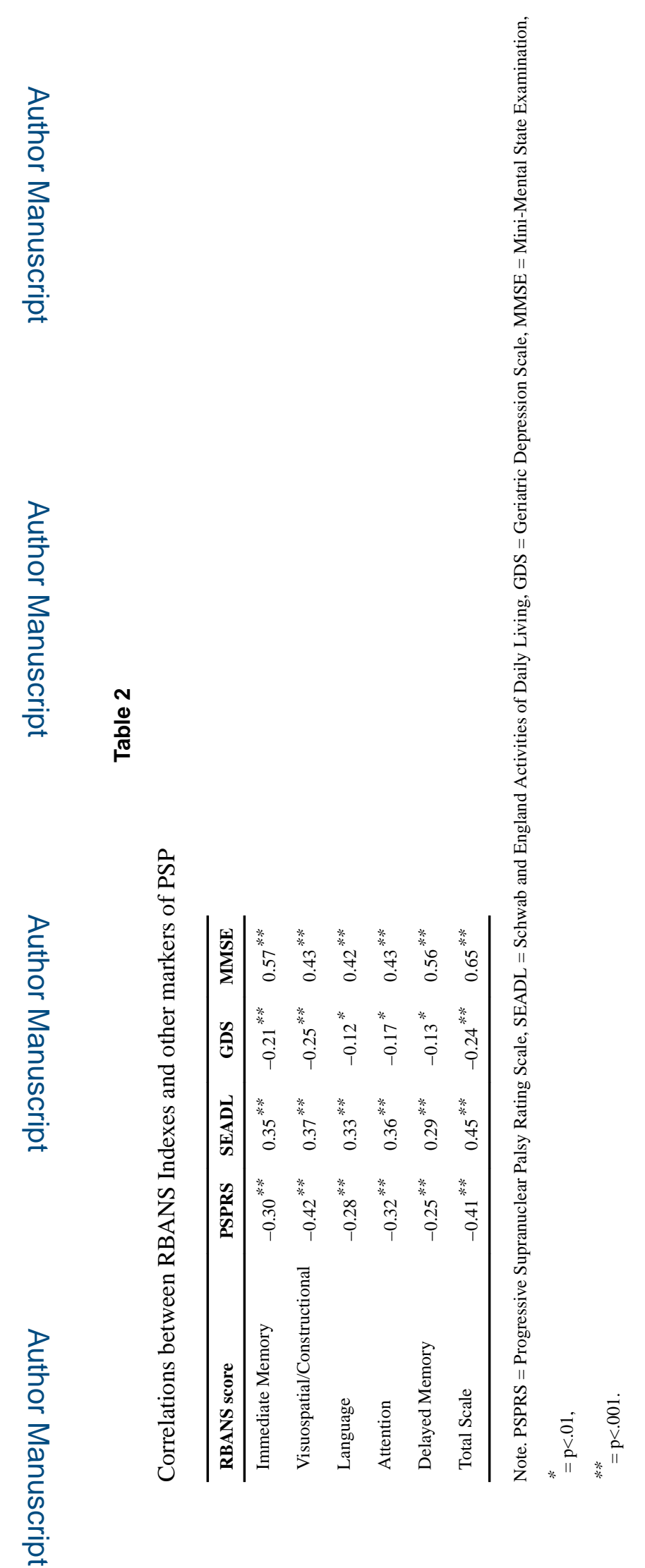

J Clin Exp Neuropsychol. Author manuscript; available in PMC 2020 July 01. 


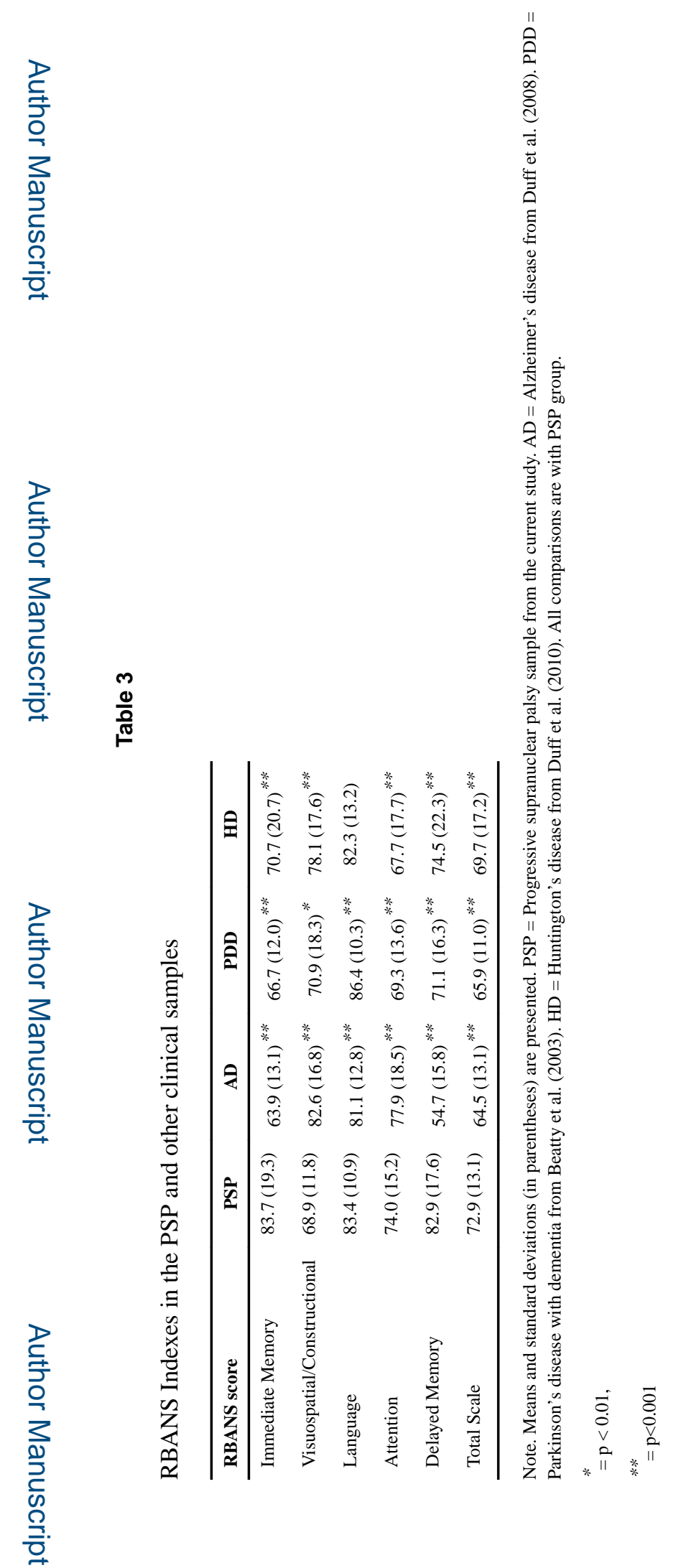

JClin Exp Neuropsychol. Author manuscript; available in PMC 2020 July 01. 